\title{
Results from systematic literature review on PE/E in Nigeria
}

\author{
Amy Dempsey \\ Population Council \\ Karen Kirk \\ Population Council
}

Follow this and additional works at: https://knowledgecommons.popcouncil.org/departments_sbsr-rh

Part of the Demography, Population, and Ecology Commons, Family, Life Course, and Society Commons, International Public Health Commons, Maternal and Child Health Commons, and the Women's Health Commons How does access to this work benefit you? Let us know!

\section{Recommended Citation}

Dempsey, Amy and Karen Kirk. 2017. "Results from systematic literature review on PE/E in Nigeria," Ending Eclampsia Country Brief. Washington, DC: Population Council. 

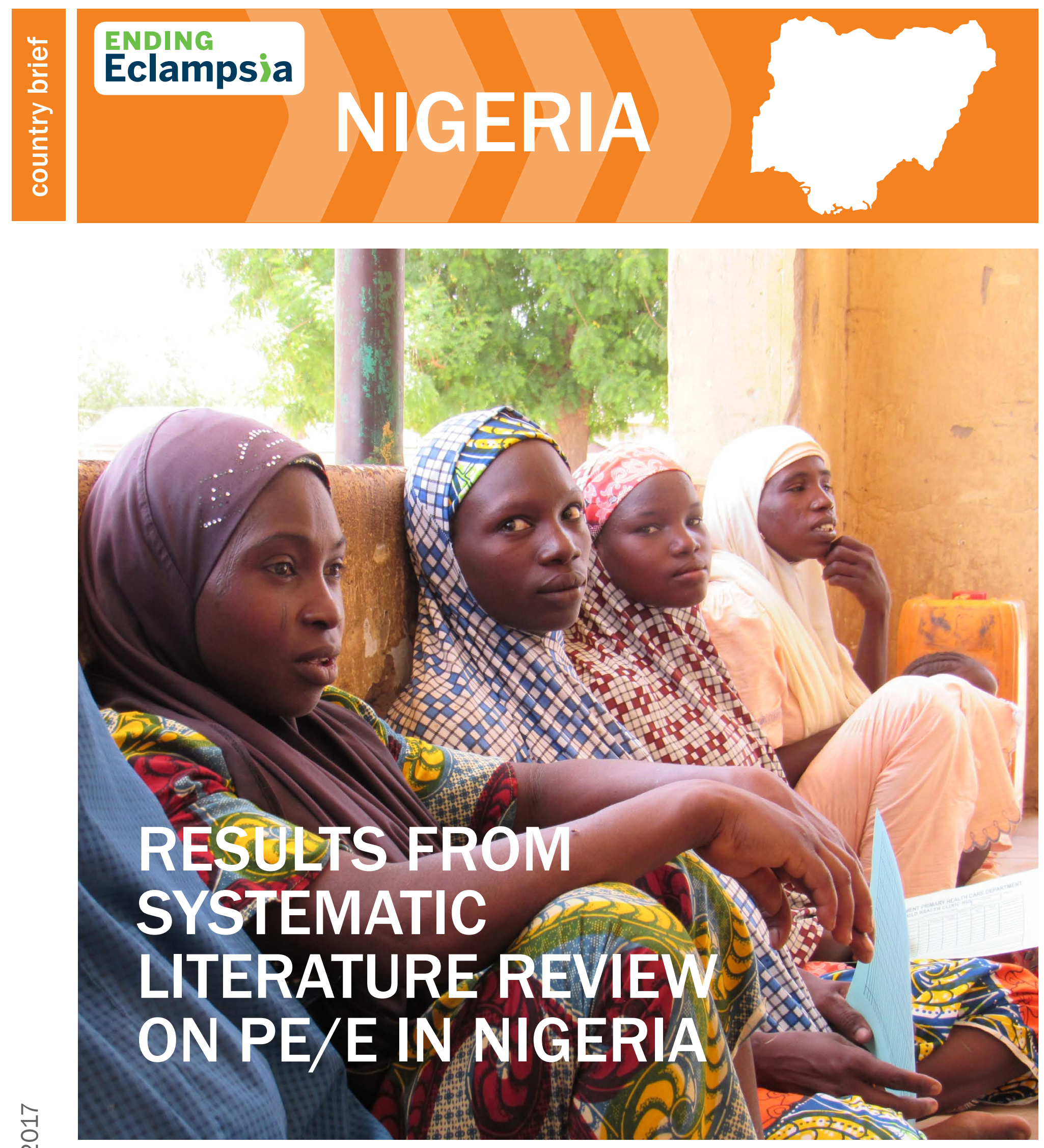

Photo credit: Karen R. Kirk, Population Council

\section{(S) USAID}

Made possible by the generous support of the American people through the United States

Agency for International Development (USAID)
The Population Council conducts research and delivers solutions that improve lives around the world. Big ideas supported by evidence: It's our model for global change. popcouncil.org

(c) 2017 The Population Council, Inc.

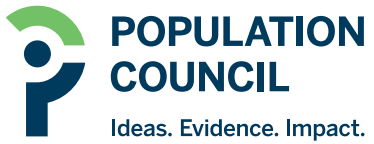




\section{BACKGROUND AND INTRODUCTION}

Despite significant declines in maternal mortality rates, sub-Saharan Africa continues to have a high burden of maternal mortality. Nigeria is one of 10 countries where women are most at risk of dying during childbirth and is responsible for 14 percent of the world's maternal deaths.

Pre-eclampsia and eclampsia (PE/E), hypertensive disorders of pregnancy, are consistently cited as a leading cause of maternal and newborn morbidity and mortality Nigeria. A recent, nationwide cross-sectional survey found that $\mathrm{PE} / \mathrm{E}$ is the leading cause of maternal mortality in Nigeria, contributing to 28.2percentofnationalmaternaldeaths. heothermaincontributorstomaternal mortality are hemorrhage, at 24.4 percent, and pregnancy-related infection or sepsis, at 14.2 percent.

To fully understand the key challenges, gaps, and interventions related to the prevention and treatment of $\mathrm{PE} / \mathrm{E}$, the Ending Eclampsia project conducted a systematic review of papers on PE/E in Nigeria published between 20002015. The methods, results, and recommendations are discussed below.

\section{METHODS}

Researchers designed two sets of key search terms related to pre-eclampsia and eclampsia to capture references for peer-reviewed papers on $P E / E$ detection, management, and prevention. The searches were run in the following bibliographic databases: PubMed, ScienceDirect, World of Science, Cochrane, POPLINE, and Wiley Online Library, as well as google.com. Two independent reviewers read and evaluated the results in three phases: Abstract and title review, full text review, and categorization of papers (Figure 1).

In Phase I, reviewers evaluated abstracts to determine relevance. Abstracts were excluded if they were:

1. Not about Nigeria;

2. Not related to pregnancy (e.g. abstracts about male subjects, children, epilepsy, and other disorders); or

3. Not related to PE/E or any associated risk factors, symptoms, or complications.

The reviewers reconciled their results, and came to a consensus on any discrepancies.

In Phase II, to confirm the results the reviewers read the full texts of the remaining citations to determine relevance and eliminated papers that were not related to $\mathrm{PE} / \mathrm{E}$, did not primarily focus on Nigeria, or did not provide any substantive data specific to Nigeria, and any papers without a full text.

Finally, the remaining articles were sorted into five categories-based on the main topic of each-that were found during the systematic review.

1. Strategies that explored prevention or managment of PE/E;

2. Demonstrated and potential risk factors for pre-eclampsia;

3. Other health outcomes associated with PE/E;

4. Descriptive program summaries and literature reviews; and

5. Record reviews of hospital case files.

The goal was to identify articles with evidence on interventions that addressed the prevention, diagnosis, and treatment of PE/E.

\section{$\mathrm{PE} / \mathrm{E}$ in Brief}

- Pre-eclampsia is a condition in pregnant women marked by an increase in blood pressure and protein in urine after 20 weeks gestation.

- Providing high quality antenatal care improves the prevention and early detection of pre-eclampsia and can prevent its progression to eclampsia.

- Eclampsia is a life-threatening condition characterized by convulsions in women with pre-eclampsia.

- Women in developing countries are 300 times more likely to die from eclampsia than women in developed countries.

- Prescribing low-dose aspirin and calcium to at-risk women can lower their risk of developing pre-eclampsia and eclampsia.

- Severe pre-eclampsia can be managed by administering anti-hypertensive drugs and magnesium sulphate $\left(\mathrm{MgSO}_{4}\right)$.

- $\mathrm{MgSO}_{4}$ is the safest and most effective treatment for severe $\mathrm{PE} / \mathrm{E}$.

- Pre-eclampsia and other hypertensive disorders in pregnancy increase the risk of preterm births, which can lead to low birth weight, anemia, and stunting.

- Improved prevention, increased detection, and effective treatment of $\mathrm{PE} / \mathrm{E}$ can prevent unnecessary maternal and newborn deaths.
The Ending Eclampsia project seeks to expand access to proven, underutilized interventions and commodities for the prevention, early detection, and treatment of pre-eclampsia and eclampsia and strengthen global partnerships. 
FIGURE 1: Flow Chart of Review Process

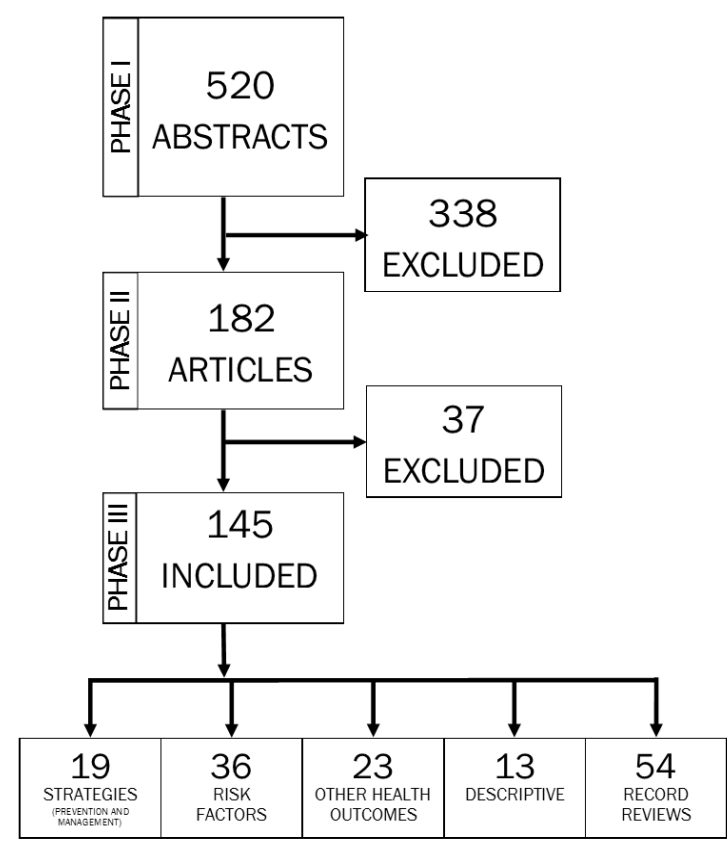

\section{RESULTS}

After removing duplicate citations and those unrelated to this review, 520 abstracts were included in Phase I. The reviewers removed 338 articles that were deemed to not fulfill the inclusion requirements for Phase I. During Phase II, 37 articles were removed as they did not provide enough, relevant Nigeria-specific information or if a full text document could not be located. The systematic review included 145 articles.

Five major themes emerged from the reviewed articles: Strategies for prevention and treatment of $P E / E$, demonstrated and potential risk factors, other health outcomes associated with $\mathrm{PE} / \mathrm{E}$, program descriptions and literature reviews, and hospital record reviews.

\section{Strategies for prevention and management}

Nineteen papers discussed strategies for detecting, monitoring, and managing PE/E. The overarching findings revealed effective case management occurs continually, from detection to after delivery. Early management of PE (before 30 weeks), using detailed monitoring charts that allow providers to document patients' health and treatment ${ }^{4}$, and institutionalizing updated policies and guidelines improve of quality care for women with $P E / E$.

Another strategy is early management of PE, incorporating a tool that streamlines monitoring and treatment, and another tests the role of serum magnesium levels in eclamptic patients as a way to test for $\mathrm{MgSO}_{4}$ toxicity.

Four articles questioned the standard dosing regimen for $\mathrm{MgSO}_{4}$ and suggested that the favored Pritchard Regimen - four grams MgSO4 IV, five grams in each buttock, followed by five grams in atlernate buttocks for 24 hours - may be excessive, and proposed that reducing the amount or frequency of doses may be as effective and safe as the currently-accepted standard.

The literature indicates that cesarean section (CS), conducted using spinal anesthesia, is likely the safest mode of delivery for PE/E cases. However, if treatment is successful, a woman can still delivery her baby vaginally.

While each of these strategies can potentially reduce mortality due to $P E / E$, the most essential strategy focuses on improving providers' knowledge and skills for detecting and managing obstetric emergencies, including task shifting to primary facility providers.

\section{Demonstrated and potential risk factors}

While the biological mechanism for developing preeclampsia remains unknown, many studies looked at various attributes or conditions in an effort to confirm or identify potential risk factors for developing PE/E. Previously demonstrated risk factors for PE/E that appear in the literature include low parity, young age, multiple gestation, history of chronic high blood pressure (BP), previous $\mathrm{PE}$, and no antenatal care (ANC) visits.

Most of the articles investigated potential risk factors relating to biomarkers in pregnancy. Various biological measures were examined to determine if there were observable trends or associations between the biomarker and the development of PE/E. The literature seems to indicate that elevated levels of haptoglobin, malondialdehyde, phenylalanine concentration, blood lead and homocysteine levels may contribute to the development of PE/E. Similarly, low serum concentrations of zinc, copper, manganese and magnesium, and low plasma calcium, vitamin $\mathrm{C}$, and $\mathrm{E}$ could also indicate preeclampsia or risk of pre-eclampsia in pregnant women.

Other potential risk factors explored in the literature were change in paternity, maternal ethnicity, and sickle cell anemia, though many studies were unable to draw firm conclusions due to small sample sizes.

\section{Other health outcomes}

Women who experience $\mathrm{PE} / \mathrm{E}$ are at increased risk of cesarean section (CS), cardiac complications, and chronic hypertension. Among the less-common outcomes reported, there were orofacial injuries and loss of vision. One article named paralysis as an outcome for one patient, but did not provide clear etiology.

Another outcome of PE/E is HELLP syndrome, a dangerous condition that complicates eclampsia with hemolysis, elevated liver enzymes, and low platelets. HELLP syndrome causes internal organs to shut down, and if left untreated, the mother's and fetus' health, will deteriorate and can lead to death. In addition to the immediate maternal health outcomes, other newborn health outcomes associated with PE/E are preterm birth, low birth weight, fetal malnutrition, and low Apgar scores. 


\section{DISCUSSION}

Despite the wealth of information in the literature on PE/E in Nigeria, gaps remain and require further research.

\section{Gaps in Prevention of PE/E}

Globally, aspirin and calcium are thought to have a protective effect for women at risk of $\mathrm{PE} / \mathrm{E}$. This is briefly mentioned without supportive evidence on the incidence of $P E / E$. The effectiveness and impact of prophylactic aspirin and calcium are unknown in the Nigerian context.

\section{Gaps in Detection of PE/E}

Gaps affecting detection of PE/E include unclear terminology and clinical definitions and low antenatal care (ANC) attendance. Even when ANC is given, providers and families are unaware of signs and symptoms of PE/E.

There is no consistency in terminology used or in clinical definitions for those terms; each paper provides their own definitions with some overlapping diagnosis criteria for BP levels, proteinuria and other symptoms including edema, headache, and visual disturbances.

The literature shows patients "booked" for ANC are less likely to develop PE/E compared to "unbooked" women but what the literature doesn't show are details about which services women receive during ANC and whether or not they are counselled on danger signs in pregnancy.

\section{Gaps in Management of PE/E}

The literature supports use of $\mathrm{MgSO}_{4}$ to manage severe pre-eclampsia and eclampsia, but the lowest effective dose is unknown. The current literature omits the utility of antihypertensive drugs to manage high BP in pregnant women, which is a strategy to reduce the risk of stroke.

Despite the evidence demonstrating the safety and effectiveness of $\mathrm{MgSO}_{4}$, its use remains low due to poor availability, inadequate knowledge among communities , lack of guidelines on its use (or unawareness of these guidelines), misinformation regarding who can administer the drug, poor knowledge of health workers on its use, fear of toxicity, little incentive for pharmaceutical companies to commercialize the drug (no return on investment), availability of less effective drugs (like diazepam), and lack of support for policy change all contribute to the underutilization of this safe and effective life-saving drug.

In addition to the relatively low use of $\mathrm{MgSO}_{4}$, there are no studies that demonstrate the lowest effective dose to manage PE/E. Some researchers have started questioning and testing the dosage with preliminary results indicating that a reduced dose-either in amount delivered or timing of doses-can be as effective as the generally-accepted Pritchard regimen.

\section{CONCLUSION AND RECOMMENDATIONS}

Throughout the development of a pregnancy, there are many opportunities when PE/E can be prevented, detected and managed; however, based on the literature, certain gaps exist along this continuum of care.

In order to have the greatest impact, we recommend a multi-level set of interventions that increase community awareness of symptoms of PE/E and ANC health seeking behavior, improve and expand provider skills and knowledge at multiple levels (including primary health centers) for detecting and treating high BP in pregnancy and $P E / E$ throughout the developmental stages of pregnancy.

To reduce the mortality associated with PE/E in Nigeria, the global community and national stakeholders must:

Develop a common language and streamlined policies:

- Develop simple terminology and associated definitions for global use.

- Advocate to hospital administrators and relevant policymakers to ensure adherence to management protocols and procurement of essential drugs.

Clarify and define management strategies and dosage:

- Determine minimum effective dose for $\mathrm{MgSO}_{4}$.

- Create and distribute clear and concise guidelines and protocols for managing hypertension and $\mathrm{PE} / \mathrm{E}$ with antihypertensives and $\mathrm{MgSO}_{4}$.

- Assess the impact of aspirin and/or calcium prophylaxis in Nigeria.

Increase access to quality services:

- Train and maintain healthcare providers on recognizing $\mathrm{PE} / \mathrm{E}$, managing high $\mathrm{BP}$ with antihypertensive drugs, when and how to administer $\mathrm{MgSO}_{4}$, and how to monitor for and treat $\mathrm{MgSO}_{4}$ toxicity.

- Train PHC providers to detect and provide initial treatment (antihypertensives and $\mathrm{MgSO}_{4}$ loading dose) for PE/E and refer to secondary facilities.

Engage Community:

- Increase community awareness and access to ANC.

- Educate community members on signs and symptoms of PE/E and when and where to seek treatment.

For the full report, visit www.endingeclampsia.org Suggested citation: Kirk, Karen R. \& Ishita Chattopadhyay. 2015. "A systematic review of the treatment and management of pre-eclampsia and eclampsia in Nigeria." Washington DC: Population Council. 